\title{
Lighting Effects on Human Behaviour and Movements: a Correlation between Space Syntax and Modelling Index in a Museum Environment
}

\author{
Giacomo Salvadori ${ }^{1}$, Davide Lista ${ }^{1}$, Francesco Leccese ${ }^{1}$, Carla Balocco ${ }^{2}$ \\ ${ }^{1}$ University of Pisa, School of Engineering, Pisa, Italy \\ ${ }^{2}$ University of Florence, School of Engineering, Florence, Italy
}

\begin{abstract}
The aim of the paper is to find a correlation between lighting parameters and spatial properties in a museum environment to understand how artificial lighting influences visitor movements and behaviours. Lighting parameters are represented by the modelling index while spatial properties by space syntax indices. The importance of tools to understand the influence of light on the visitor experience in a museum is very important because lighting is crucial in designing art exhibitions. The results obtained by correlations in galleries of the Natural History Museum of the University of Pisa (Italy) suggest that human behaviour is more influenced by the visual interaction between different points of the space than by the shortest paths linking different points.
\end{abstract}

\section{Introduction}

The purpose of the paper is to assess the efficacy of space syntax as a simulation tool for lighting designers and establish a relation between spatial properties and artificial lighting parameters in a museum environment. Spatial properties are investigated through a space syntax analysis, a verified methodology to forecast human behaviours and movements in spatial layouts (Balocco, 2018). Among lighting parameters, special relevance has the modelling index because is able to describe how a display set is perceived: the appearance of an object is enhanced when its features are lit so that form and textures are revealed clearly and pleasingly, meaning that light should not be too directional, or it produces harsh shadows, neither should be too diffuse, or the contrast is null (EN 12464-1, 2011). The aim is to investigate and understand how artificial lighting influence visitor movements in a museum environment. The comprehension of the effects due to lighting is essential in exhibitions design and in enhancing the visitors experience. In a museum contest, lighting is one of the most powerful curatorial tools available to fulfils the main goal of a museum as a space for contemplating art (Feltrin, 2017; Leccese, 2018; Krukar, 2014). Space syntax analysis gives a theoretical understanding on how people make use of space and if a correlation between space syntax indices and lighting parameters is found, the result is that light also contributes to influence people behaviours and movements. Providing lighting designers with simulation tools to understand and control the influence of light upon the experience of the visitors is an important goal because lighting in museum takes a leading part in affecting the visitor behaviour and is a crucial step when designing art exhibitions. An effective and efficient method for design different lighting strategies (for the enhancement of observer perception, preferences and patterns of their visual scanning) is provided by means of space syntax analysis and correlation indices.

Space syntax analysis has been applied to urban studies and complex buildings. In the first case, it has been used as a method for street lighting rationalization, energy saving improvement, outdoor environmental quality and lighting systems sustainability (Beccali, 2018; Choi, 2006; Kazemidemneh, 2018; Leccese, 2019). In the second case, it has been used as a method for interior spatial structure rearrangement, for user wayfinding evaluation and for user safety and orientation, i.e. in offices, shopping malls, hospitals, museums, railway stations and cultural buildings (Antonakaki, 2007; Tzortzi, 2011). At the present status in the scientific literature, space syntax has already been applied to carry out studies in interior environments of museums. For example in 2003, Stavroulaki and Peponis analysed with space syntax the spatial construction of Carlo Scarpa's design of the Castelvecchio art museum at Verona (Stavroulaki, 2003). In 2012, Reynoso and Reynoso studied the best places for effective security control at the Museo Nacional de Colombia (Reynoso, 2012). In 2014, Krukar analysed the spatial organisation of museums and its influence on the visitors experience (Krukar, 2014). At present, there are no literature references concerning the use of space syntax for improving lighting in indoor environment (enhancing visitors experience and visual perception) and for protecting artworks by preventive conservation.

In this paper the space syntax methodology is described and applied to the case study of the Natural History Museum of the University of Pisa, where some galleries were analysed. The obtained results are discussed with special reference to the correlation between spatial properties and modelling index. The modelling index can be considered as the lighting parameter most influencing the spatial perception of the exibited artworks.

\section{Case study: the Natural History Museum of the University of Pisa}

The Natural History Museum of the University of Pisa is one of the most ancient museums in the world. The 
Museum was born at the end of the XVI century as a "Gallery" attached to the Pisa Garden of Simples (the current Botanic Garden). Since the end of the Seventies, the Museum is hosted in the Carthusian Monastery of Calci (near Pisa), a XIV centuries building of great historical-architectural value (see Figure 1). In Figure 2, the ground, the first and the second floor plans of the galleries hosting the Museum are shown.

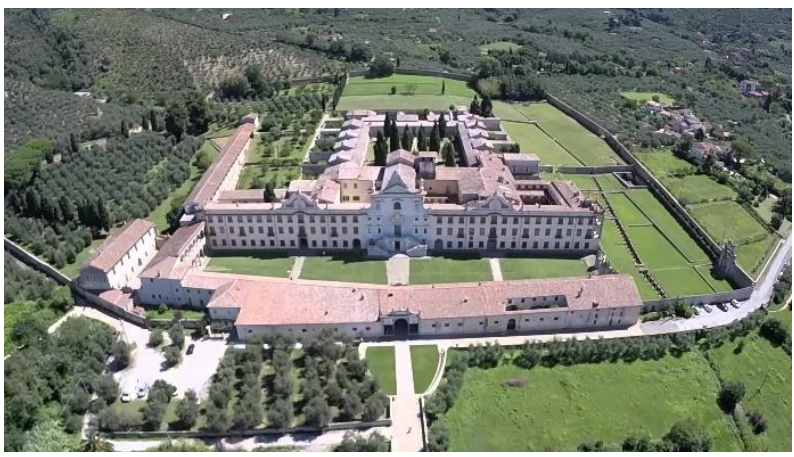

Figure 1: Aerial view from the west of the Carthusian Monastery that houses the Natural History Museum.

\section{Space syntax applied to indoor environments}

In order to evaluate the possibility to use space syntax analysis to improve lighting and enhance visual experience, this type of technique was applied to some galleries of the Natural History Museum.

\section{Space syntax analysis background}

Space syntax analyses are a complex of techniques for the analysis of the inter-visibility connections within buildings or urban networks.
The analyses are based on the role of the space configuration as the primary element generating the movement flows that occur on it (Turner, 2005). In the late 1980s Hillier and Hanson published the book "The Social Logic of Space" (Hillier, 1988), in which they discussed the existence of a two-way relationship between space and socio-cultural models; spatial syntax theory is a method to explore this relationship (Kazemidemneh, 2018). The theory states that movement is essentially related to the space configuration and is its major product (Dursun, 2007).

The most used space syntax techniques are the axial analysis, the angular segment analysis and the visibility graph analysis (Bebhabani, 2014).

The axial analysis assumes that urban space is articulated into a plot of lines. Each straight line corresponds to a human sight line: it is assumed that an observer perceives the space of the city by straight lines corresponding to his perspectives and his movement in the city is guided by them (Sharmin, 2017).

The angular segment analysis is an extension of the axial analysis. In angular segment analysis, the axial lines are broken up into segments, from junction to junction. What the angular segment analysis adds to is that each segment is weighted by the angle of its connections to other segments (Turner, 2000; Kolovou, 2017).

The visibility graph analysis is a technique based on the concept that a user moves in space in base of how he perceives the space. Therefore, the basic element of visibility graph analysis is the single point. Space is divided in a cluster of points and every point is identified as the possible location of the user (Lee, 2017).

Second floor
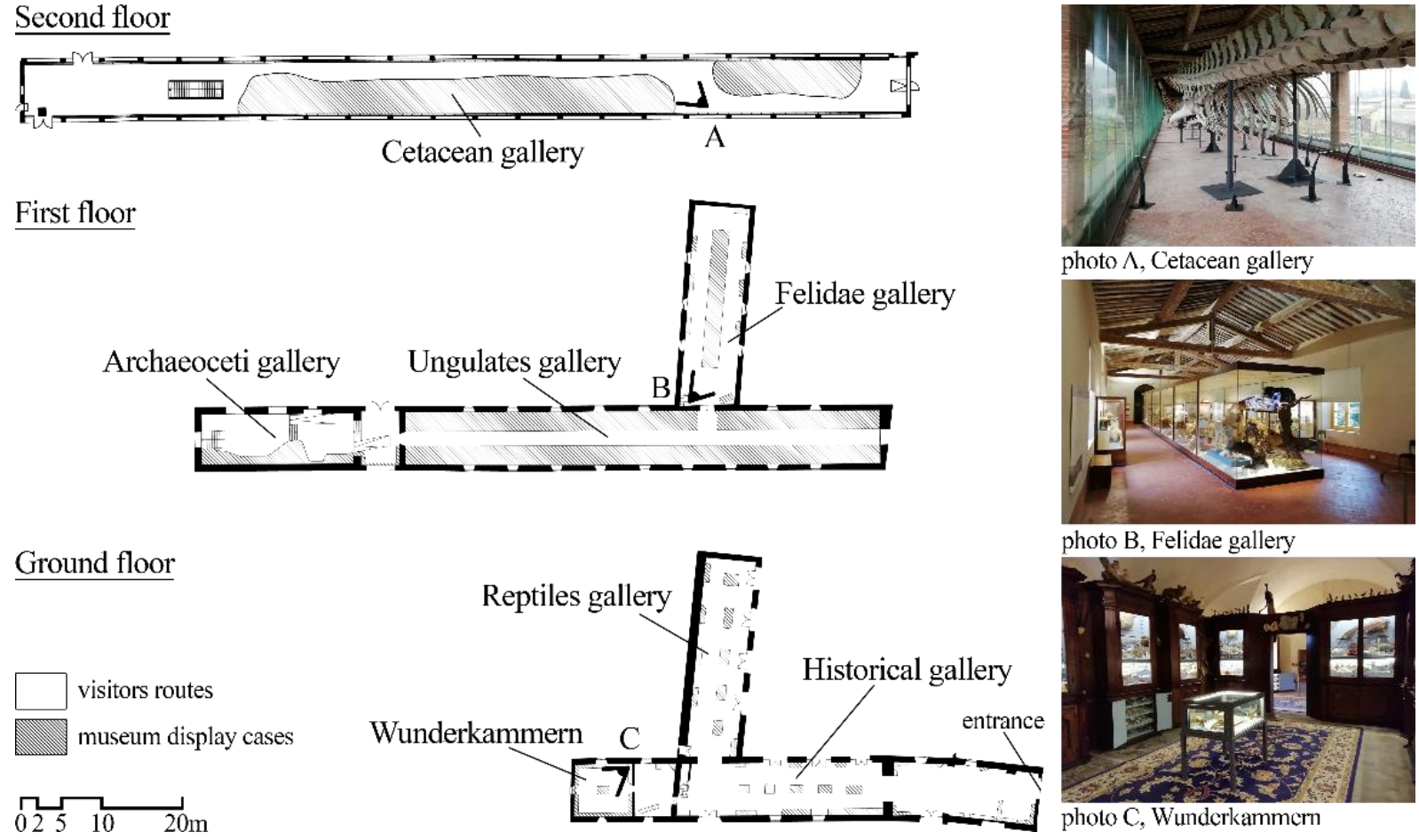

Figure 2: Plans of the galleries of the Natural History Museum of the University of Pisa (Italy). 
Among the various available techniques for space syntax analysis, the visibility graph analysis was chosen, deemed more suitable for building interiors because is suited to spaces with defined areas and clear boundaries. In the visibility graph analysis, each point of the space has a unique geometrical relationship with its surroundings which gives it unique visual properties. In a plan, a unique property of each point is the area visible from that point, in the shape of a polygon, called isovist (van der Hoeven, 2014). Due to the impracticality of considering all points, the space is typically articulated into a fine grid (ideally in the size of a human) and isovists of each cell on the grid are drawn. A graph is then developed with the cells as its nodes and the existence of visibility between cells as its edges (Turner, 2001). The properties of the isovists have already been linked with human spatial behaviour by putting in evidence how humans are sensitive to the isovist area and quite skilled at recognising the points in space which provide the largest isovist size (Wiener, 2007).

\section{Syntactic measures assessment}

After superimposing a grid on the analysed spatial layout, different numeric values (syntactic measures) is possible to calculate to give a quantitative value to the obtained representation. Among the various syntactic measures, the connectivity $(C)$ and the integration $(I)$ indices have been used. The connectivity index $(C)$ counts how many points of the grid are visible from an analysed point, given the walls restrictions. The connectivity index derives from the concept of isovist, which is a polygon drawn on a building layout around a point of reference (Benedict, 1979). This polygon covers the entire surface area visible from the reference point (see Figure 3). The integration index (I) represents the accessibility of a given point within the analysed layout and describes the mean depth of a point with respect all the other points (Cataldo, 2015). The depth of a single point is defined as the topological (and not geometrical) distance separating a pair of points and it is measured as the number of points that divides them along the shortest path linking the two points.
The integration index $(I)$ is defined as:

$$
I=(k-1) / D_{t}
$$

with: $k$ total number of points in the grid superimposed to the spatial layout, $D_{t}$ total depth of a point (the total depth of a point is the sum of the depths with respect all the other points). The integration index quantifies how close a given point is from all the other points. The greater the integration index value of a point in a spatial layout is, the more accessible and integrated in the system is, vice versa the lower the value of the integration index is, the more isolated and segregated the point results.

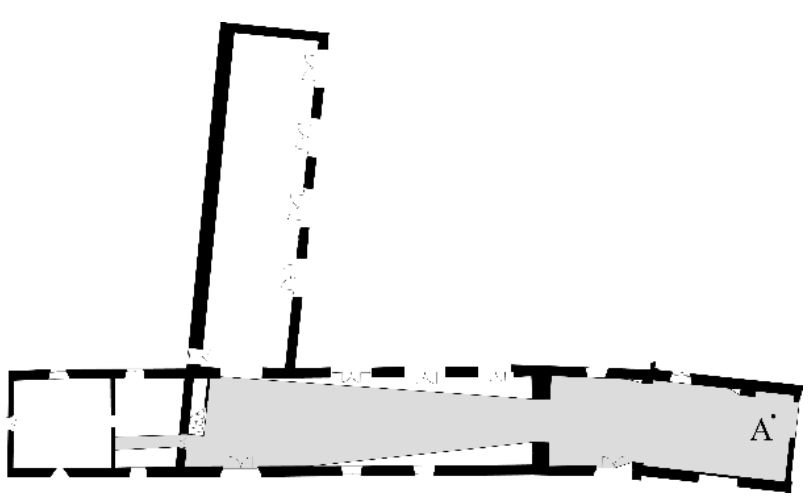

Figure 3: Example of the isovist polygon for the point A, corresponding to the entrance of the museum at the ground floor.

\section{Space syntax analysis results of the case study}

Space syntax analysis can be automated with the help of softwares, in this paper the spatial properties have been derived using depthmapX software (UCL-Bartlett School of Architecture-Space Syntax Lab). The input data for the software are the plans of the museum galleries in a vector format ( $d x f$ file). In the plans, the boundaries given by walls and museum display cases are reported to reproduce the viewer's eye limits (at the height of $1.50 \mathrm{~m}$ ). The outputs of the software are colour charts and numeric tables, and are very useful for the comparison of the various syntactic measures produced.
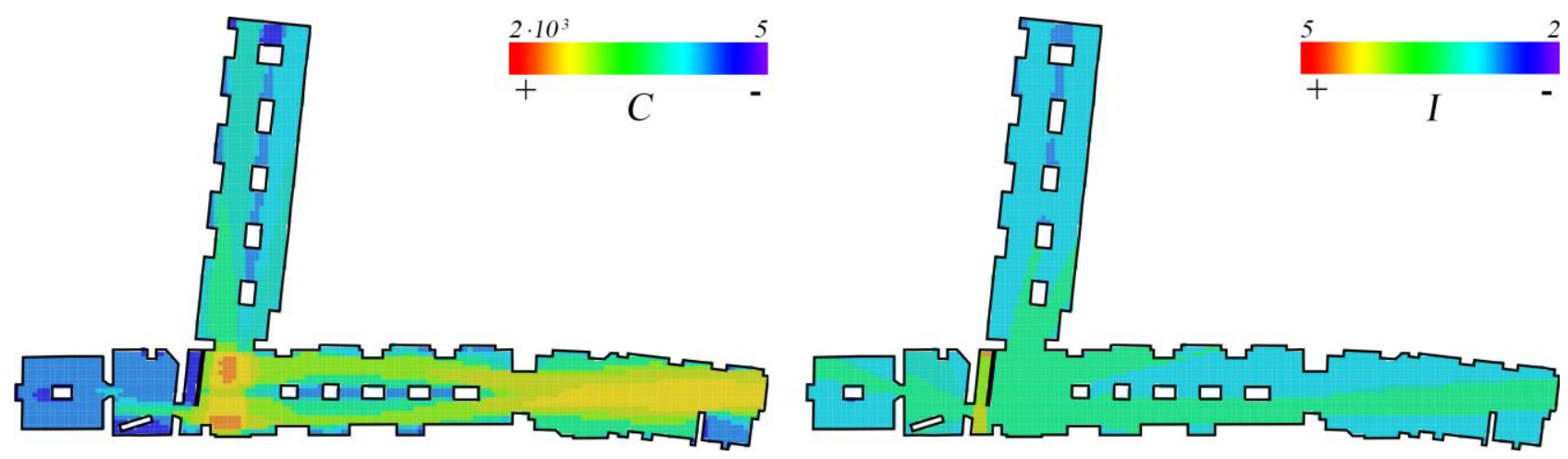

Figure 4: Visibility graph analysis for the ground floor of the Natural Museum of the University of Pisa: (left) connectivity index (C), (right) integration index (I) chromatic scales. 
As an example in Figure 4, the visibility graph analysis for the museum ground floor galleries, chosen as the case study, is shown. In Figure 4 the syntactic measures of the connectivity index (on the left) and of the integration index (on the right) are shown with a chromatic scale. The colour scale, according to (Turner, 2004), runs from blue (very low values) to red (very high values).

In the space syntax analysis, the three floors have been placed next to each other. If the floors were placed above the others, the analyses and building simulation would have been more complex. In the latter case, a more advanced tool set working in three-dimensions would have been required (while depthmapX is a software for two-dimensions analyses). To work around the threedimension problem, links have been added manually. Two links have been manually added between the ground floor and the first floor and between the first floor and the second floor at the position of the stairs.

At the end of the space syntax analysis, applied to the three floors of the Museum, the results are the following. The minimum value obtained for the connectivity index (C) is 6 , the maximum value is 2056 ; the minimum value obtained for the integration index (I) is 1.71, the maximum value is 4.66 .

The highest values of the connectivity index (C) are at the entrance of the Museum, at the intersection between the Historical gallery and the Reptiles gallery (ground floor, Figure 2) and at the top of the stairs in the Cetacean gallery (second floor, Figure 2). The lowest values of the connectivity index are in the Wunderkammern (ground floor, Figure 2) which has a space configuration as a cul-de-sac.

The highest values of the integration index (I) are for the points near the stairs leading from the ground floor to the first (see Figure 2). These points are the most barycentric in the Museum layout, so the most central and integrated points. The lowest values of the integration index are for the points at the end of the Cetacean gallery (second floor, Figure 2). These points are the most segregated and peripheral in the Museum layout.

\section{The modelling index}

The appearance of a building interior is enhanced when the structural features, the people and the objects within the space are lit so that form and texture are revealed clearly and pleasingly. This feature is very important in museum galleries where the action of seeing object is the goal and the main purpose of visitors, so the visual task is at the base of the visitor experience.

In order to have objects lit in a clearly and pleasingly way, lighting should not be too directional or it will produce harsh shadows, neither should it be too diffuse or the modelling effect will be lost entirely, resulting in a boring luminous environment. According to the European technical standard and international guidelines, the modelling index should be considered to evaluate the modelling effects for both indoor and outdoor environments.
The modelling index was chosen among the various lighting parameters to be related to the spatial properties of the museum galleries, where the visitor looks at the exhibited objects walking along a path (with short stops) with a behaviour similar to that held in outdoor pedestrian paths. For this reason the modelling index $(M)$, introduced by CIE $136 / 2000$ and defined as the ratio of vertical $\left(E_{V}\right)$ to semi-cylindrical $\left(E_{S C}\right)$ illuminance at a point, was chosen:

$$
M=E_{V} / E_{S C}
$$

For uniform arrangement of luminaires or roof lights, a value of $M$ in the range $0.8-1.3$ is an indicator of good modelling. In other words, people consider the lighting of 3D-objects features to be well balanced when the modelling index is included in this range of values (Zaikina, 2016). The modelling effect improves as the modelling index decreases. For values of the modelling index higher than 1.3, shadows appear very soft and the 3D-objects contour are not clearly distinguishable from the background. For values of the modelling index lower than 0.8 , the perceived images are with a high contrast and shadows are very harsh.

In this paper the modelling index was experimentally evaluated by a survey campaign and calculated by software simulations, with the aim to point out possible correlations between spatial properties and modelling index.

\section{In situ measures of the modelling index}

A survey campaign of the modelling index was carried out in the Historical gallery of the Museum, without the contribution of daylighting. Usually, different series experimental measurements on lighting measurements need an adequate measurement grid set up. In Figure 5, the experimental grid adopted in the Historical gallery is shown. In Table 1 , the measured values of the semicylindrical $\left(E_{S C}\right)$ and vertical $\left(E_{V}\right)$ illuminance are shown. At each point of the measurement grid, the modelling index was calculated as the ratio between the semi-cylindrical and the vertical illuminance in the main direction in which the space is travelled by the visitors.

Table 1: Illuminance values measured in the Historical gallery (the IDs of the anlysed points are indicated in Figure 5).

\begin{tabular}{ccc|ccc}
\hline $\begin{array}{c}\text { Points } \\
\text { ID }\end{array}$ & $\begin{array}{c}\boldsymbol{E}_{\boldsymbol{S C}} \\
(l x)\end{array}$ & $\begin{array}{c}\boldsymbol{E}_{\boldsymbol{V}} \\
(l x)\end{array}$ & $\begin{array}{c}\text { Points } \\
\text { ID }\end{array}$ & $\begin{array}{c}\boldsymbol{E}_{\boldsymbol{S C}} \\
(l x)\end{array}$ & $\begin{array}{c}\boldsymbol{E}_{\boldsymbol{V}} \\
(l x)\end{array}$ \\
\hline 1 & 62.8 & 54.5 & 10 & 54.2 & 63.9 \\
2 & 55.1 & 38.9 & 11 & 51.8 & 52.9 \\
3 & 52.4 & 46.2 & 12 & 68.7 & 66.6 \\
4 & 48.8 & 27.4 & 13 & 52.3 & 30.5 \\
5 & 66.7 & 33.4 & 14 & 40.3 & 31.5 \\
6 & 35.2 & 23.1 & 15 & 51.5 & 41.3 \\
7 & 28.2 & 18.7 & 16 & 20.9 & 15.5 \\
8 & 15.5 & 15.7 & 17 & 13.1 & 13.6 \\
9 & 11.5 & 11.9 & & & \\
\hline
\end{tabular}




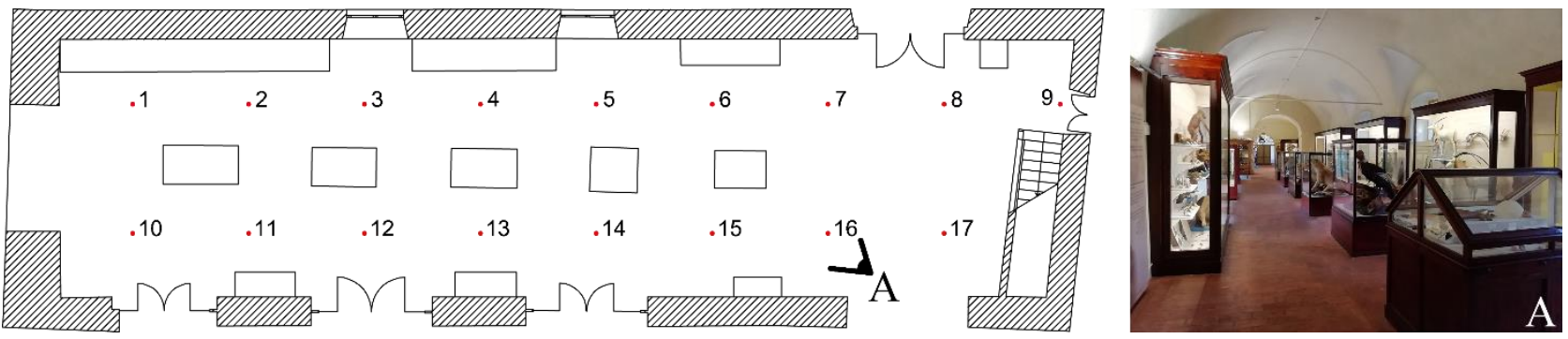

Figure 5: Historical gallery, grid of the illuminance measurement points (left) and photo of the room (right).

All the instruments used, belong to the Laboratory of Lighting and Acoustics of the School of Engineering (University of Pisa). The technical instruments characteristics are in compliance with the requirements suggested in UNI 11142 Italian technical standards (UNI 11142, 2004). For the vertical illuminance measurements the luxmeter Delta Ohm mod.2101.2 was used, equipped with the adequate probe mod.LP 471 PHOT. For the semy-cilindrical illuminance measurements the luxmeter PRC Krockmann mod.Radiolux 111 was used, equipped with the adequate probe mod.111112-2. The values of semi-cylindrical and vertical illuminance have been measured at a height of $1.50 \mathrm{~m}$. The used instruments are accompanied by the calibration certificates, issued by accredited laboratories, according to the Italian standard for portable photometers (UNI 11142, 2004).

\section{Software simulation of the modelling index}

Simulations were carried out by DIALux evo software (DIAL Gmbh), using a 3D model of the Historical gallery and luminaires arrangements, without the contribution of daylighting. Photometric data of the luminaires were implemented using EULUMDAT photometric files. In Table 2, the luminaire main photometric data are shown.

Table 2: Photometric data of the luminaire installed in the Historical gallery of the Museum.

$\int \frac{\frac{P=31 \mathrm{~W}}{\Phi=2240 \mathrm{~lm}}}{\frac{\eta}{\eta=72 \mathrm{~lm} / \mathrm{W}}} \frac{\mathrm{T}_{\mathrm{C}}=3000 \mathrm{~K}}{}$

In Figure 6, the luminaire position is provided. Luminaires in the Historical gallery are 9 and are mounted at a height of $3.20 \mathrm{~m}$. Simulations were performed by estimating the maintenance factors according to CIE 97 recommendation (CIE 97, 2005).

In Table 3, the illuminance simulated values for the same points of the measurement grid are shown. The simulation model was validated comparing the obtained calculated values with in situ measured ones. The percentage difference between the modelling indices calculated from the measured values and the modelling indices calculated from software simulations are always less than $14 \%$.

In Figure 7, a false-colours rendering of the vertical illuminance mapping and semi-cylindrical illuminance mapping for the Historical gallery are shown, at the height of $1.50 \mathrm{~m}$.

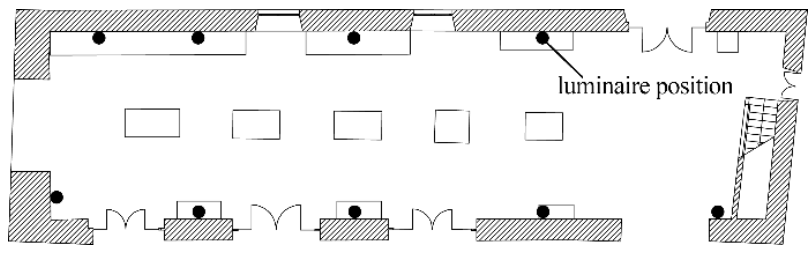

Figure 6: Luminaire arrangements in the Historical gallery.

Table 3: Illuminance values simulated in the Historical gallery (the IDs of the anlysed points are indicated in Figure 5).

\begin{tabular}{ccc|ccc}
\hline $\begin{array}{c}\text { Points } \\
\text { ID }\end{array}$ & $\begin{array}{c}\boldsymbol{E}_{\boldsymbol{S C}} \\
(l x)\end{array}$ & $\begin{array}{c}\boldsymbol{E}_{\boldsymbol{V}} \\
(l x)\end{array}$ & $\begin{array}{c}\text { Points } \\
\text { ID }\end{array}$ & $\begin{array}{c}\boldsymbol{E}_{\boldsymbol{S C}} \\
(l x)\end{array}$ & $\begin{array}{c}\boldsymbol{E}_{\boldsymbol{V}} \\
(l x)\end{array}$ \\
\hline 1 & 64.7 & 47.8 & 10 & 61.6 & 63.3 \\
2 & 59.2 & 42.4 & 11 & 58.2 & 54.5 \\
3 & 50.4 & 41.6 & 12 & 66.7 & 60.5 \\
4 & 54.2 & 34.5 & 13 & 52.8 & 35.0 \\
5 & 58.5 & 34.1 & 14 & 44.3 & 33.9 \\
6 & 37.0 & 23.3 & 15 & 45.6 & 39.3 \\
7 & 30.6 & 19.5 & 16 & 21.3 & 15.2 \\
8 & 14.5 & 14.8 & 17 & 12.5 & 14.8 \\
9 & 11.3 & 10.9 & & & \\
\hline
\end{tabular}

\section{Research of correlation between spatial properties and modelling index}

The purpose of the paper is to assess the efficacy of space syntax as a simulation tool for lighting designers and establish a relation between spatial properties and lighting parameters in a museum environment. For this reason, in this Section a relationship between the space syntax indices and the modelling index was investigated. The correlation was researched in 17 points, the same of the measurement grid shown in Figure 5. 

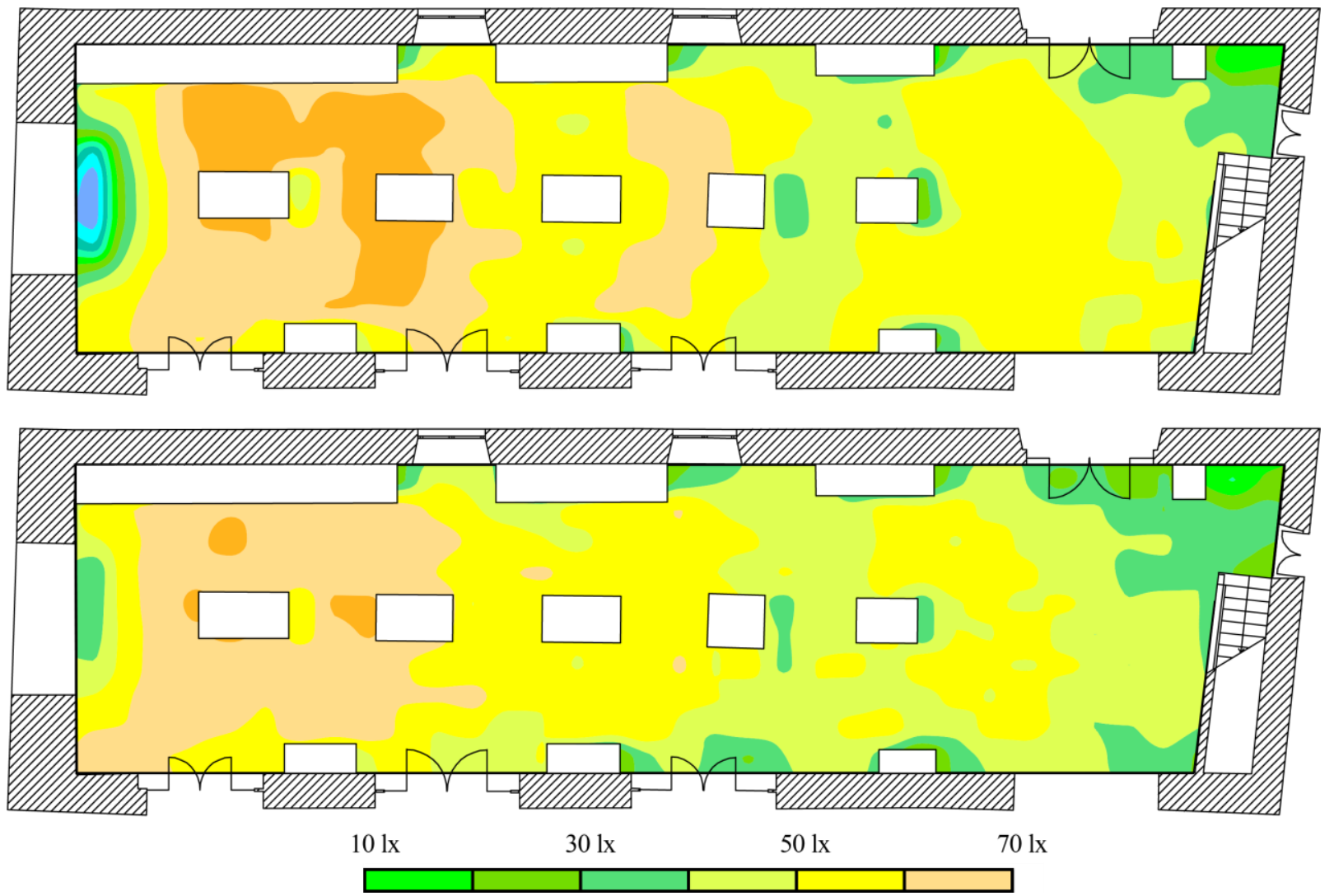

Figure 7: False-colours rendering of the vertical illuminance mapping (top) and semi-cylindrical illuminance mapping (bottom) for the Historical gallery.

Relations between the space syntax indices and the modelling index were carried out with a linear regression analysis performed using the least squares method and then calculating the coefficient of determination $\left(R^{2}\right)$. The least squares method is an optimization technique with the aim to find an interpolation function that is as close as possible to the points of a data set. The coefficient $R^{2}$ indicates which fraction of the data variance is due to the linear dependence between the variables $x$ and $y . R^{2}$ indicates how much the linear regression model is adherent to the phenomenon under study. That is, the more $R^{2}$ is close to 1 , the greater the goodness-of-fit of the linear model is.

Two cases were analysed. Within the first one correlations between space syntax indices and modelling index were investigated. Within the second one correlations between the space syntax indices and the natural logarithm of the modelling index were investigated.

\section{Correlations between space syntax indices and modelling index}

In the case of the Historical gallery of the Museum: in Figure 8, the correlation between the integration index and the modelling index is shown; in Figure 9, the correlation between the connectivity index and the modelling index is shown.
In the correlation between the integration index and the modelling index (see Figure 8), the coefficient $R^{2}$ is near to zero and the regression line cannot express the trend of the data that appears disordered and chaotic. As a matter of fact, data are very scattered with respect to the regression line. The horizontal regression line suggests that for the variations of the integration index are not influencing the modelling index variations.

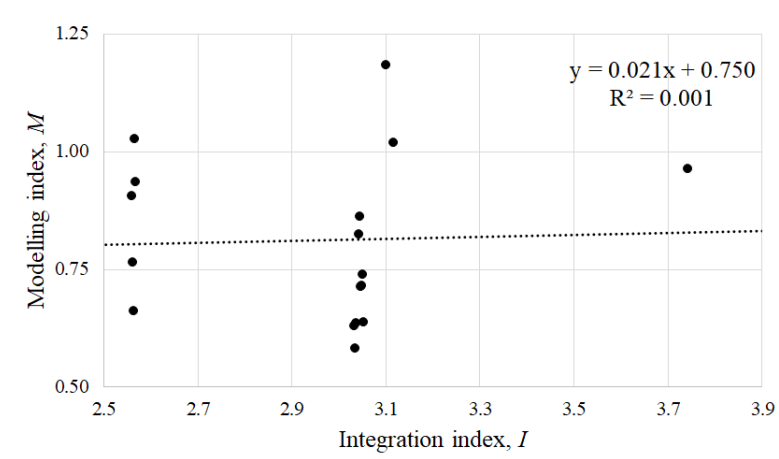

Figure 8: Correlation between the integration index (I) and the modelling index $(M)$ for the Historical gallery.

The coefficient $R^{2}$ provided in Figure $9\left(R^{2}=0.70\right)$ shows that the regression line can explain the data trend with $70 \%$ probability. 


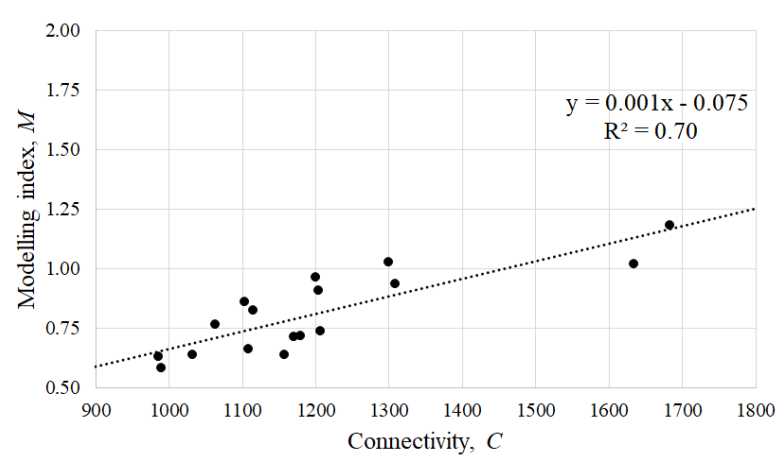

Figure 9: Correlation between the connectivity index $(C)$ and the modelling index (I) for the Historical gallery.

\section{Correlations between the space syntax indices and the natural logarithm of the modelling index}

In this case, referred to the Historical gallery of the Museum again, the use of the natural logarithm was adopted according to (Nenci, 2006). In Figure 10, the correlation between the integration index and the logarithm of the modelling index is shown. In Figure 11, the correlation between the connectivity index and the logarithm of the modelling index is shown.

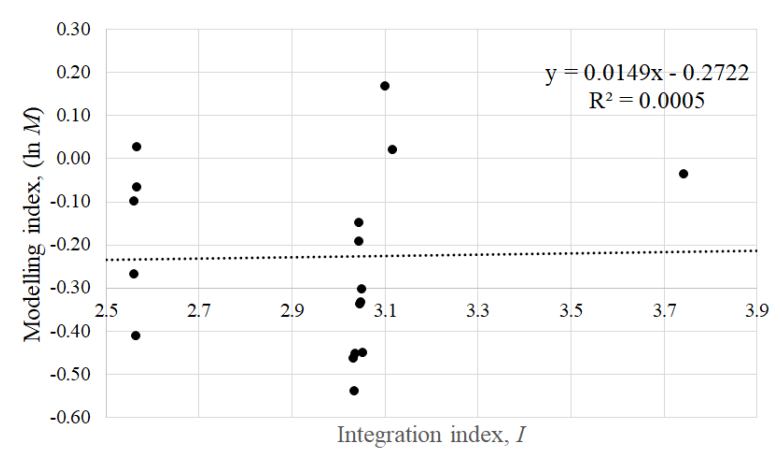

Figure 10: Correlation between the integration index (I) and the logarithm of the modelling index $(M)$ for the Historical gallery.

In Figure 10, the coefficient $R^{2}$ of the correlation between the integration index and the logarithm of the modelling index is near to zero as in the previous case (see Figure 8). All the data appear very scattered with respect to the regression line.

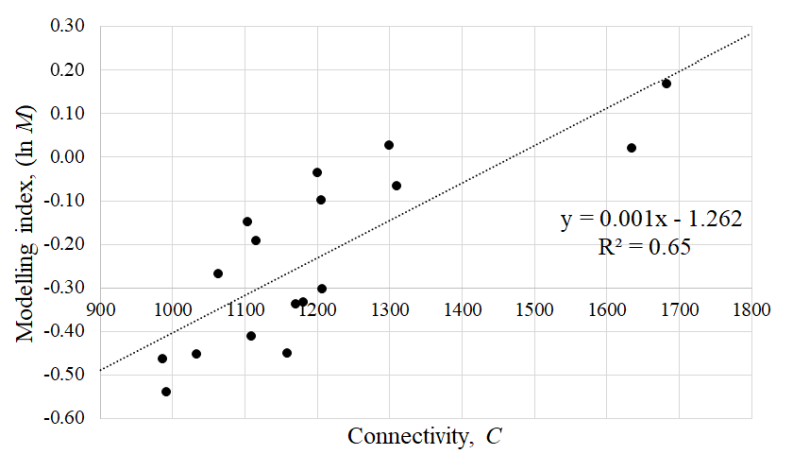

Figure 11: Correlation between the connectivity index $(C)$ and the logarithm of the modelling index (M) for the Historical gallery.
The coefficient $R^{2}$ provided in Figure $11 \quad\left(R^{2}=0.65\right)$ shows that the regression line can explain the data trend with $65 \%$ probability. In the comparison of correlations between the connectivity index and the modelling index, the coefficient $R^{2}$ moderately increases from 0.65 (present case, see Figure 11) to 0.70 (previous case, see Figure 9) and the slope of the regression line is always positive.

\section{Discussion and conclusive remarks}

The analysis discussed for the Historical gallery of the Natural History Museum of the University of Pisa shows an important correlation between the modelling index and the space syntax connectivity index (the correlation was expressed by the $R^{2}$ coefficient showing a maximum value of 0.70$)$. The correlation refers to standard conditions of artificial lighting as experienced by visitors.

For all the analysed rooms of the Museum, the correlation with the integration index $(I)$ was found to be negligible on the contrary the correlation with the connectivity index $(C)$ was found stronger. These results suggest that in museum spaces human behaviour is more influenced by the visual connections and the visibility interactions (meaning the property, expressed by the connectivity index, of a point to be visible to all other points in space) than by the shortest paths linking different points (property expressed by the integration index).

The correlation found shows a link between human behaviours and movements and lighting levels, confirming the initial hypothesis. Understanding the more crowded area or which are the focal points of a space can help lighting designers in redirecting lights for the desired effects.

This research is a preliminary study to explore the potential of space syntax as a tool to understand the influence of lighting on human behaviours. The results appear encouraging, but an extensive research in a large number of cases, considering also the effects of daylighting (in our present study only artificial light was considered), could reinforce the discussed outcomes.

\section{Acknowledgements}

This research was partially funded by the University of Pisa within the plurennial project: 'Recognition of the current state and in-depth research for the conservation and enhancement of the monumental buildings of the Certosa di Calci and its Museums' (starting date: April 2017), under the supervision of the Ministry of Cultural Heritage.

\section{References}

Antonakaki, T. (2007). Lighting and spatial structure in religious architecture: a comparative study of a Byzantine church and an early Ottoman mosque in the city of Thessaloniki. $6^{\text {th }}$ International Space Syntax Symposium. Istanbul (TR), 12-15 June 2007.

Balocco, C. and Volante, G. (2018). Lighting design for energy sustainability, information and perception. A 
museum environment as a case study. Sustainability $10,1671-1688$

Bebhabani, P.A., Gu, N., Ostwald, M.J. (2014). Comparing the properties of different space syntax techniques for analysing interiors. $48^{\text {th }}$ International Conference of the Architectural Science Association. Genova (I), 10-13 December 2014.

Beccali, M., Bonomolo, M., Leccese, F., Lista, D., Salvadori, G. (2018). On the impact of safety requirements, energy prices and investment costs in street lighting refurbishment design. Energy 165, 739-759.

Benedict, M.L. (1979). To take hold of space: isovists and isovist fields. Environment and Planning B 6 , 47-65.

Cataldo, A., Di Pinto, V., Rinaldi, A.M. (2015). Representing and sharing spatial knowledge using configurational ontology. International Journal Business Intelligence and Data Mining 10, 123-151.

Choi, A.S., Kim, Y.O., Oh, E.S., Kim, Y.S. (2006). Application of the space syntax theory to quantitative street lighting design. Building and Environment 41, 355-366.

CIE 136 (2000). Guide to the lighting of urban areas. Commission Internationale de l'Éclairage.

CIE 97 (2005). Guide on the maintenance of indoor electric lighting systems. Commission Internationale de l'Éclairage.

Dursun, P. (2007). Space syntax in architectural design. $6^{\text {th }}$ International Space Syntax Symposium. Istanbul (TR), 12-15 June 2007.

EN 12464-1 (2011). Light and lighting - Lighting of work places - Part 1: Indoor work places. European Committee for Standardization.

Feltrin, F., Leccese, F., Hanselaer, P., Smet, K. (2017) Analysis of painted artworks' color appearance under various lighting settings. $17^{\text {th }}$ IEEE International Conference on Environment and Electrical Engineering. Milan (I), 6-9 June 2017.

Hillier, B., Hanson, J. (1988). The social logic of space. Cambridge University Press. Cambridge (UK).

van der Hoeven, F., van Nes, A. (2014). Improving the design of urban underground space in metro stations using the space syntax methodology. Tunnelling and Underground Space Technology 40, 64-74.

Leccese, F., Lista, D., Salvadori, G., Beccali, M., Bonomolo, M. (2019). Space syntax analysis applied to urban street lighting: Relations between spatial properties and lighting levels. Applied Sciences 9(16), Article number 3331, 1-24.

Leccese, F. Salvadori, G. Feltrin, F. Morozzi, R. Nieri, P. (2018). Study on the suitable lighting design of Beato Angelico's artworks displayed at the National Museum of San Matteo in Pisa (Italy). IOP Conference Series: Materials Science and Engineering 364(1), 1-8.
Lee, J.H. Ostwald, M.J. Lee, H. (2017). Measuring the spatial and social characteristics of the architectural plans of aged care facilities. Frontiers of Architectural Research 6, 431-441.

Kazemidemneh, M. Mohammadjavad, M (2018). Use of space syntax technique to improve the quality of lighting and modify energy consumption patterns in urban spaces. European Journal of Sustainable Development 7, 29-40.

Kolovou, I. Gil, J. Karimi, K. Law, S. Versluis L. (2017). Road centre line simplification principles for angular segment analysis. $11^{\text {th }}$ Conference on Space Syntax. Lisbon (P), 3-7 July 2017.

Krukar, J. (2014). Walk, look, remember: the influence of the gallery's spatial layout on human memory for an art exhibition. Behavioral Sciences 4, 181-201.

Nenci, A.M. Troffa, R. (2006). Integrating space syntax in wayfinding analysis. Workshop on space syntax and spatial cognition. Bremen (D), 24 September 2006.

Reynoso, C. Reynoso, P. (2012). Space syntax in museum exhibition security and scripting effects: the Museo Nacional de Colombia. Proceedings from the $8^{\text {th }}$ International Space Syntax Symposium. Santiago de Chile (RCH), 3-6 January 2012.

Sharmin, S. Kamruzzaman, M. (2017). Meta-analysis of the relationship between space syntax measures and pedestrian movement. Transport and review, 1-27.

Stavroulaki, G. Peponis, J. (2003). The spatial construction of seeing at Castelvecchio. $4^{\text {th }}$ International Space Syntax Symposium. London (UK), 17-19 June 2003.

Turner, A. (2000). Angular analysis: a method fo the quantification of space. UCL. London (UK).

Turner, A. (2001). From isovists to visibility graphs: a methodology for the analysis of architectural space. Environment and Planning B: Plannings and Design 25, 103-121.

Turner, A. (2004). Depthmap $4-A$ researcher's handbook. UCL-Bartlett School of Graduate Studies. London (UK).

Turner, A. Penn, A. Hillier, B. (2005). An algorithmic definition of the axial map. Environment and Planning B: Plannings and Design 32, 425-444.

Tzortzi, K. (2015). Spatial concepts in museum theory and practice. $10^{\text {th }}$ International Space Syntax Symposium. London (UK), 13-17 July 2015.

UNI 11142 (2004). Light and lighting - Portable photometers - Performance requirements. Ente Nazionale Italiano di Unificazione.

Wiener, J.M. Franz, G. Rossmanith, N. Reichelt, A. Mallot, H.A. Bülthoff, H.H. (2007). Isovist analysis captures properties of space relevant for locomotion and experience. Perception 36, 1066-1083.

Zaikina, V. (2016). Light modelling in architectural spaces. Doctoral Thesis. NTUN. Trondheim (N). 